\title{
APL functions for interactive data analysis: Graphics and labels
}

\author{
SELBY EVANS, JERRY D. NEIDEFFER, and FRED H. GAGE \\ Texas Christian University, Fort Worth, Texas 76129
}

\begin{abstract}
APL functions designed to provide labeled plots and histograms are described. Support functions that augment a data file with necessary information to label output and to maintain a common plotting scale are also described. APL code and illustrative output are presented.
\end{abstract}

This report presents APL functions designed to produce graphic displays of data and to support the labeling of analyses using data files. The plotting functions provide histograms and scatter plots of raw data. Included is a basic plotting function that can be used to meet almost any plotting requirements for which typed output is adequate.

The labeling functions facilitate the use of APL files to maintain data for analysis. Files make it possible to work with a much larger data base than can be accommodated in a work space. Moreover, the data set is better protected from inadvertent change. With the labels included in the data file, functions can access both data and labels at the same time, without user attention and without risk of user error in associating labels with results. For plotting, a standard scale can also be established for each variable, permitting data subsets to be plotted on the same scale.

\section{SUPPORT FUNCTIONS: LABELS AND SCALE CONTROL}

Functions designed to prepare a file for use with plotting functions are given in Figure 1. These support functions incorporate into the file information needed to maintain common scales for the plots and to label the results. The benefits of labeled results, of course, are not limited to plots.

\section{LABL}

LABL augments a file composed of data tables by appending three components. The first additional file component is a user-supplied title intended to appear as an identifying header on all results derived from the file. The second additional file component is a scale table, described under the function TSCALE. The last, a table of contents, is a two-dimensional array with rows carrying column and table names. Note that LABL assumes that the file to be augmented is tied to the number 1 and open for both read and write (as done by the standard file-tying function, FTIE). LABL is primarily intended to be called by a user interface function that would make the tie for it. This use is illustrated in the function GRAPHICS, described in a later section of this report.
The table of contents carries the column names as the first $\mathrm{N}$ rows, where $\mathrm{N}$ is the number of columns in the data tables. (It is assumed that all tables have the same number of columns.) Row N+1 is blank, to serve as a separator; the remaining rows carry the table names.

The names supplied by LABL are necessarily general: $\mathrm{T} 1, \mathrm{~T} 2$, and so on, for tables. We find it convenient to reserve the first column for subject numbers and the second column for a number designating a relevant category, such as experimental condition or subject attribute. Therefore, LABL assigns the names ITEMS and SETS to the first two columns and then begins variable names, V1, V2, and so on, in Column 3. Interpreting the output is facilitated by revising the table of contents to carry column and table names relevant to the research. This revision can easily be done by hand in the desk calculator mode. The standard table of contents permits use of functions that assume a table of contents without demanding that the user prepare one. Note how the use of LABL in the interface function, GRAPHICS, allows the user to go directly from a set of raw data tables in a file to the initial investigation of plotted results.

\section{$S \leftarrow$ TSCALE F}

TSCALE operates on a file containing one or more tables to produce a reasonable plotting scale for each column. The file is assumed to be tied to the number 1 . The right argument, $\mathrm{F}$, indicates the highest file component number to be included in setting the scale. TSCALE assumes that file components numbered by the integers 1 through $F$ are to be used. This condition will be met if the user starts with an empty file and appends the tables.

The result, $\mathrm{S}$, contains the completed scale table as a 2 by $\mathrm{N}$ array, where $\mathrm{N}$ is the number of columns. The first row gives the minimum values for each column; the second row gives the maximum values. To use the scale table in conjunction with SPLOT, the user or function selects the appropriate columns in the scale table and stores them in the variable SCL. To avoid work-space full problems with a large number of variables, TSCALE will process only the first 40 columns. In psychological research, data sets with more than 40 columns are usually psychological measuring instruments 


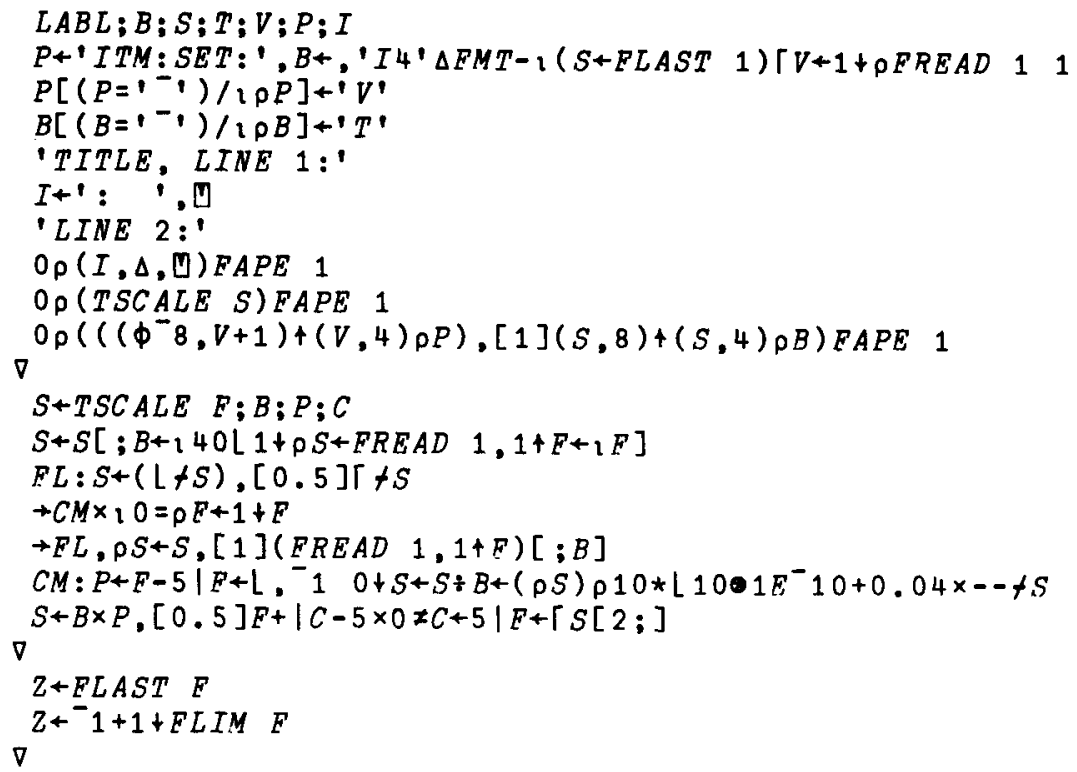

Figure 1. APL code for support functions LABL, TSCALE, and FLAST.

in which the same scale is appropriate for all items. In that case, a function can apply the scale from Column 40 to all columns with numbers greater than 40 , as is done by GRAPHICS.

TSCALE determines the scale for each column by first finding the minimum and maximum values for each column, taken across tables. It may then extend the range slightly so that the limits are multiples of 5 or 10 when expressed in units appropriate for the observed range. We find that the resulting scales are generally satisfactory for plots, but the user can easily change the stored values if necessary. The array is stored in the second of the three file components added by LABL.

TSCALE is useful in working with a file containing several data sets in which the columns represent the same variables. The data sets, as tables, might represent successive stages of training or different groups of subjects. In such cases, plotting all data sets on the same scale is quite useful in comparing or presenting the results.

\section{T $\leftarrow$ FLAST F}

FLAST gives the number of the last component in the file tied to the number $F$. It is a minor but convenient modification of the standard file function FLIM, providing directly the result that must frequently be computed from FLIM.

\section{PLOTTING FUNCTIONS}

Plotting functions are given in Figure 2. The first, SPLOT, is a basic function that can be used to meet many different plotting needs. The second, GRAPHICS, meets one such need, providing convenient support for graphic display of raw data in the form of plots and histograms. Figure 3 illustrates the results of GRAPHICS.

\section{G $\leftarrow$ L SPLOT D}

SPLOT is a general-purpose plotting function with several features designed to assist in interactive data analysis. The right argument, $\mathrm{D}$, is an array of two or three columns. Each row corresponds to a point to be plotted. Column 1 gives the X-coordinate and Column 2, the Y-coordinate. The third column, if supplied, specifies the plotting symbol for that point. If the third column is not supplied, SPLOT will provide a common plotting symbol for all points.

The left argument, $\mathrm{L}$, carries two values that determine, in order, the number of rows and columns in the output plot (excluding the area devoted to scales for the axes). The result, $G$, is a character vector that will reproduce the plot.

SPLOT requires a global variable, $S C L$, to specify the ranges for each axis. SPLOT will compute the values itself if SCL is specified as a scalar zero. The new ranges will be left in SCL.

SCL is a two-dimensional array with columns corresponding to $\mathrm{X}$ - and $\mathrm{Y}$-axes, respectively. The top row gives the minimum values for each axis; the bottom row gives maximum values. SPLOT will extend the range of SCL to accommodate points that lie beyond this range and give the notice "SCL REVISED." Since the value of the global SCL is altered, the change remains available for the user to consult as an indication of the ranges that should have been used. If several plots are made and revision of SCL is left up to SPLOT, the final values of SCL will accommodate all the data sets that were plotted.

The use of the global SCL to control ranges makes it possible to plot multiple sets of data to the same scale. It is necessary only to determine a plotting range that will accommodate all data sets. TSCALE will determine this range automatically for data in a file. 


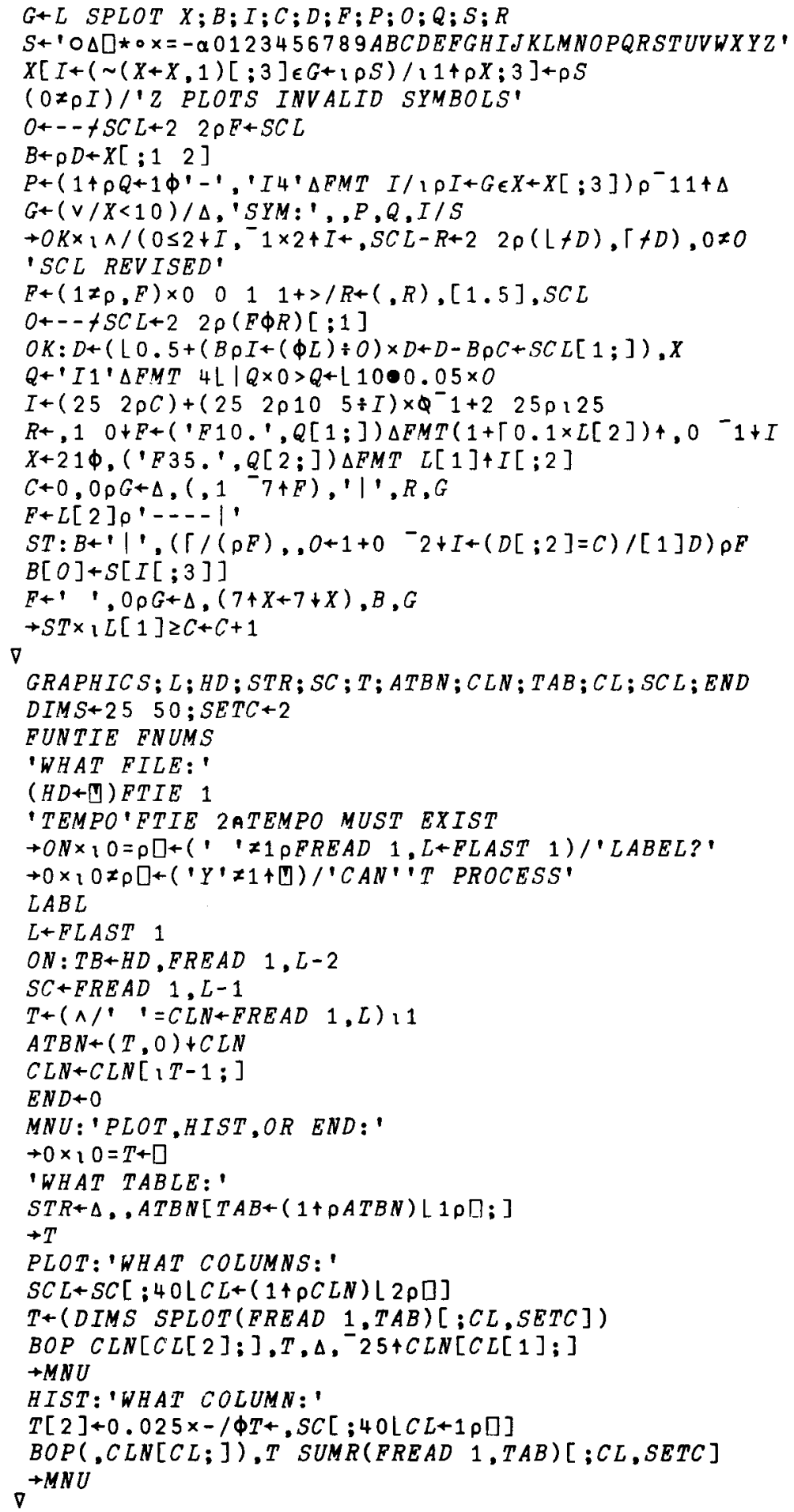

Figure 2. APL code for plotting functions SPLOT and GRAPHICS. Note that GRAPHICS ties the data file to 1 and the output file, TEMPO, to 2 for use by the support functions that it calls.

The entries in Column 3 should be positive integers less than 46. (Values that do not meet this requirement will be plotted with the symbol Z.) The entries in Column 3 will select plotting symbols in the corresponding location in the character vector specified in Line 1 of SPLOT. The user may substitute other characters in this vector without consequence for the rest of the function.

Plotting symbols corresponding to individual subject or item numbers (less than 36) can be produced by adding 10 to the numbers and using the result as Column 3. The plotted symbols will run from 1 to 9 
GRAPHICS

WHAT FILE:

GRAFILE

$L A B E L$ ?

$Y E S$

TITLE, LINE 1:

APL GRAPHICS

LINE 2:

PLOTTING DEMONSTRATION

PLOT,HIST,OR END:

$\mathrm{Q}$ :

PLOT

WHAT TABLE:

Q:

1

WHAT COLUMNS:

:

56

1

PUSH RET

GRAFILE: APL GRAPHICS

PLOTTING DEMONSTRATION

$T 1$

$C T R=0.19$

$V_{4}$

1.01

01

$\Delta$

$\Delta$

$\Delta$

$\Delta$

$\Delta$

$\Delta$

$\Delta$

$\Delta$

$\Delta$

$\Delta$

$$
\Delta
$$

$\Delta$

$\Delta$

$\Delta$

$\Delta$

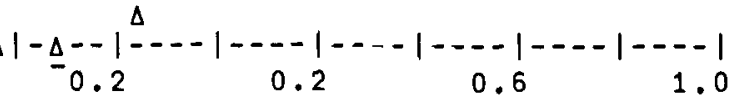

V3

PLOT, HIST, OR END:

C:

END

Figure 3. Illustration of the use and output of the user interface function GRAPHICS. GRAFILE contained one table and was unlabeled when GRAPHICS was invoked. The function requested authorization to label the flle and then did so. Data were generated by CORGEN (Evans, Gage, \& Neideffer, 1980). To conform to the conventions described in the text, two new columns were catenated in front of the data array produced by CORGEN. These became Columns 1 and 2 of the data table. Column 1 carried the integers 1-30, representing item numbers. Column 2 carried the value 2 in each row. That value selected the plotting symbol, as indicated on the plot. 
and continue with $\mathrm{A}, \mathrm{B}, \mathrm{C}$ to represent double-digit numbers.

SPLOT will ordinarily provide, below the plot, a mapping of plotting symbols into the numbers in Column 3. If all the numbers are greater than 10 , this output is suppressed on the assumption that the resulting numbers and letters are being used to represent individual subjects or items known to the user. In this usage, the mapping list is not only redundant, it is inconveniently long.

SPLOT also adjusts its format controls so that the decimal precision in labeling an axis is consistent with the range of the axis: The smaller the range, the more decimal precision is used. This adjustment allows display of values as large as tens of thousands or as small as ten thousandths.

With $24 \mathrm{~K}$ available in the work space, SPLOT will plot about 500 points with $\mathrm{L}$ set to 2030 . With large plots, the user may note a delay of a few seconds before the plot is complete.

\section{GRAPHICS}

GRAPHICS is a user interface function designed to relieve the user of attention to the details of accessing data and recording the identity of the data sets being processed. It produces plots and histograms from a data file containing one or more tables. It supports plotting of any pair of columns in any selected table. Similarly, it provides a histogram and summary statistics for any column in any selected table. Scales for plots and histograms are controlled so that they are the same for a given column, regardless of what table it is drawn from. This arrangement is useful in comparing results on the same measure across different data sets.

The function assumes that data are stored in a file in the form of one or more two-dimensional tables, all with the same number of columns. The graphic operations assume that the file is labeled as described in the preceding section, but GRAPHICS checks for that condition and, if the file is not labeled, will invoke the process itself. Note that this check depends on the assumption that the first character of the first column label is a blank.
GRAPHICS also assumes that the data tables carry, in Column 2, integers suitable for selecting the plotting symbol for SPLOT. This column may be used to desig. nate experimental groups or categories of subjects.

The choice of Column 2 as a source of the plotting symbol designation is made in Line 1 of GRAPHICS, in the specification of SETC to the value of 2 . Some users may prefer to omit this specification and leave SETC as a global variable in the work space. That way, it can easily be revised by the user. The same comments apply to the specification of DIMS, the number of rows and columns in the plot.

GRAPHICS also provides a histogram and statistical summary for any data column. This option requires SUMR and its supporting functions (Evans \& Gage, 1979). GRAPHICS uses the general output function, BOP (Evans, Gage, \& Neideffer, 1980), which establishes output conventions. These conventions include filing the result in the temporary output file, TEMPO. Since these results are in the form of a character vector, they can be modified by REPLACES (Evans et al., 1980), or by almost any text editing function the user may find in the local APL library. The results, if printed on a typewriter-quality terminal, are usable as a final copy for some purposes.

With data arranged in files and adequately labeled, a wide variety of analytic techniques becomes feasible. The previously given CORRELATE function (Evans et al., 1980) can easily be adapted to the conventions above. Reports on principal components analysis, discriminant function analysis, analysis of variance, and file-oriented data entry are presently being prepared. These functions will be consistent with the file conventions described here.

\section{REFERENCES}

Evans, S., \& Gage, F. H. APL programs for interactive data analysis: Basic statistics and histograms. Behavior Research Methods \& Instrumentation, 1979, 11, 605-606.

Evans, S., Gage, F. H., \& Neideffer, J. D. APL programs for interactive data analysis: Data entry and correlation. Behavior Research Methods \& Instrumentation, 1980, 12, 372-375.

(Received for publication October 12, 1980; accepted October 16, 1980.) 